\title{
VIAS BILIFERAS NO TAPIR OU ANTA (Tapirus americanus)
}

\author{
THE BIIARY TREE OF THE TAPIR (Tapinus americamus)
}

Maria Angélica MI(;I,INO'; Wilson Machado de SOLZA': L,iberato Joào Afonso DIDIO'

\begin{abstract}
RESUMO
()s autores estuclaram as vias bilíferas do tapir ou anta (Tapirus americanus). após injeção do siskema excretor do fígado de 2 animais, machos e adultos, com látex Neoprene 650 corado. fixação das peças com soluçăo aquosa de formol a 10\%: e dissecação. () ductus choledocus origina-se a partir da confluência do ramus principalis dexter e do sinister. sendo este animal desprovido de vesícula biliar. () ramus principalis dexter ć formado pelos ramus ventralis lobi dextri, ramus medius lobi dextri, ramus dorsalis lobi dextri c ramus processi caudati. os quais se unem por diferentes modalidades. O ramus principalis sinister é formado pelos ramus medius lobi sinistri lateralis, ramus dorsalis lobi sinistri lateralis, ramus lobi quadrati, ramus ventralis lobi sinistri lateralis c ramus lobi sinistri medialis. com diferentes arranjos.
\end{abstract}

UNITLRMOS: Figado; Anta; Ductos biliares

\section{INTROIUÇÃO}

A distribuiçāo intraparenquimatosa das vias bilíferas do tapir ou anta (Tapirus americanus) é o objeto deste artigo, que apresenta o estudo anatomico dos elementos excretores do fígado, num animal de difícil obtenção, no qual esta glândula possui lobação semelhante à dos equíideos e de tipo intermediário entre a lobação acentuada dos carnívoros e a discreta lobaçāo dos ruminantes. Além disso, o fato do tapir estar entre os poucos animais que não possuem vesícula biliar foi outra motivação para encetar esta pesquisa para veriticar a morfologia do ducto colédoco.

Como a busca bibliográfica não registrou trabalho sobre este assunto na anta. limitamo-nos a consignar as publicações $\mathrm{cm}$ equídeos, tanto domésticos, quanto silvestres, ou seja, equiinos sem raça definida - S.R.D). (FERREIRA², 1974); muar (FERREIRA et al, ${ }^{3,45}, 1976$ ); jumento nordestino (PRADA el al. . 1982); zebra (D'ERRICO et al.', 1983); eqüinos puro sangue inglês - P.S.I. (STOPIGil IA et al. ${ }^{10}, 1986$ e MARÇAL $^{7}$, 1987). Naturalmente, estes trabalhos permitirão estudo comparativo das vias bilíferas do tapir com as dos eqüídeos.

\section{MATFRIAL, F, MÉTODO}

Foram utilizados para este estudo, lígados de 2 exemplares de Tapirus americanus, machos, adultos, oriundos do Pantanal do Estado de Mato Grosso do Sul (MS), Brasil.
Os órgãos foram obtidos durante a necroscopia dos animais com a porção do duodeno correspondente à terminação do ductus choledocus na papila duodenal maior. Através desta esvaziamos, tanto quanto possível. o sistema bilífero, para depois, também por ela, injetá-lo com Neoprene látex-650, diluído $\mathrm{cm}$ água e corado. A seguir, os órgãos foram fixados em solução de formol a $10 \%$, dissecados pela face visceral, esquematizados e fotografados. Para facilitar a exposição dos resultados c considerando que o fígado da anta se mostra incompletamente separado em lobos por incisuras, dividimos a glândula de acordo com SCHUMMER; NICKEL' ( 1979): por meio de dois planos imaginários, perpendiculares às faces diafragmática c visceral do fígado, um acompanhando o cixo maior da fissura hepática direita, até atingir dorsalmente a veia cava caudal; o outro, acompanhando a incisura correspondente ao ligamento redondo até cruzar a impressão esofágica. Assim, à direita do primeiro plano, encontra-se o lobus dexter. pequena parte de lobus caudatus e o processus caudatus. e à esquerda do segundo plano, situam-se o lobus sinister lateralis co lobus sinister medialis. A porção intermédia apresenta, ventralmente, o lobus quadratus e, dorsalmente, o lobus caudatus, correspondente à pars supraportalis. No transcorrer destas dissecações, estabelecemos as divisões das porções direita e esquerda da glândula, identificando na superfícic partes dorsal (1/4), média (2/4) e ventral $(1 / 4)$.

1-Professor Associado - Fätculdade de Medicina Veterinlíria e /enoleconla da LSP

2-Professur Adjunlo" Faculdade de Medicina Veterintiria da LiVI:SP" . "'ampus" de Araçatuba

3-Professor Visitante - Escolla Paulisla de Mcdicina 


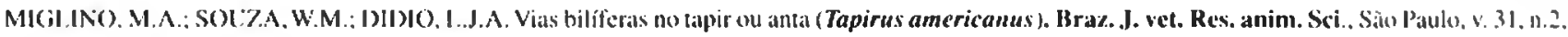
p. 95.4 .1094 .

Relativamente à sistematizaçāo dats vias bilíferas, consideramos mais a extensão dos territórios drenados do que o calibre dos dectos, pois a injeção de massia pode favorecer um melhor preenchimento dos ductos de localização superficial. Não registramos os pequenos afluentes das tributárias mais calibrosals, apenas quando drenavam as mesmas regiōes correspondentes aos mesmos, não se associando portanto, a contribuição de procedência diversa. Para efeito da descrição. obedecemos a direção de tluxo biliar, isto É. periferiacentro. quando respeitamos a identidade dos ramos nominados. alti sua atluência a outro de igual caltegoria ou a troncos resultantes de suas aferências, mesmo que por vezes inominados.

Na apresentação dos resultados, utilizamos a nomenclatura adotada por JABLAN-PANTIC (1963), ressalvando-se. cntretanto. a designação empregada para o ductus choledocus.

\section{RESLLTADOS}

O estudo das vias bilíferas dos 2 fígados de Tapirus americanus, machos, adultos, permite observar que o ductus choledocus, desprovido de afluentes nas duas glândulas. origina-sc a partir da reunião do ramus principalis dexter (d) com o ramus principalis sinister (e). O primciro destes apresenta como componentes o ramus ventralis lobi dextri. o ramus medius lobi dextri. o ramus processi caudati e o ramus dorsalis lobi dextri. Em um órgão, o segundo e o terceiro afferentes formam um tronco comum, ao qual vêm ter pela ordem o primeiro e o último. Ao ramus dorsalis lobi dextri ca a) ramus medius lobi dextri vêm ter aferentes inominados isolados do lobus dexter (território lateral e ventral). No outro fígado (Obs.2), os componentes agrupamse 2 a 2 para formar dois troncos comuns, que são alluentes independentes do lobus dexter (território ventral). Fstes são seguidos de tronco de dois elementos, um desta mesmal origem (território dorsal) e ou do lobus caudatus (processus caudalus). () ramus principalis dexter depois de formado encontra-se live de afluentes em ambas as preparaçōes.

De outra parte, o ramus principalis sinister surge, nos dois fígados analisados, constituído pelos ramus ventralis lobi sinistri lateralis, ramus lobi sinister medialis. ramus medius lobi sinistri lateralis, ramus dorsalis lobi sinistri lateralis c ramus lobi quadrati. os quais se apresentam sob dois diferentes arranjos na composição da citada via. Deste modo. em uma das peças, o ramus dorsalis lobi sinistri lateralis unc-sc ao ramus medius lobi sinistri lateralis para compor tronco comum, quando ambos estão livres de alluentes, mas o citado tronco por eles formado recebe aferente isolado do lobus caudatus (pars supraportalis). Por sua vez, o ramus ventralis lobi sinistri lateralis aflui ao ramus lobi sinistri medialis compondo tronco, oportunidade em que. enquanto o primeiro deles apresenta-se isento de eferentes, o outro recebe coletor do lobus quadratus. A viat comum aqui composta está liver de tributárias e, nesta glândulat, une-se à anteriormente descrital. paral formar ducto de drenagem que. após unir-se ao ramus lobi quadrati live de aferentes inominados, constitui já o próprio ramus principalis sinister do lobus caudatus (pars supraportalis) e o líltimo do lobus quadratus.

No outro órgão, o ramus medius lobi sinistri lateralis alsocial-se ao ramus ventralis lobi sinistri lateralis formando Ironco. Tanto estes dois coletores quanto a via por eles formada. surgem sem aferentes. O tronco aqui formado acolhe. a seguir, o ramus dorsalis lobi sinistri lateralis encontrando-se, de igual modo, tanto esta via quanto o tronco resultante de stla confluência. sem afluentes. Na sequiência. vem ter a este ducto o ramus lobi sinistri medialis. o qual recebe em seu trajeto um tribulário do lobus quadratus: após citada convergência. o colctor lormado acolhe componente inominada do lobus caudatus (pars supraportalis) para depois receber o ramus lobi quadrati, isento de alluentes nat glândula cm questão. Caraclerizamos desta forma o próprio ramus principalis sinister. que acolhe em seu percurso colctor inominado do lobus quadratus.

\section{COMENTÁRIOS}

Morfologicamente. como já afirmamos. o tïgado do tapir assemelha-se alo do equiino. chamando a altenção, alcim da ausência da vesícula hiliar. os contornos dos lobos ca as incisuras interlobares.

Notamos nas duas peças que com nallural dificuldade conseguimos obter, que o ductus choledocus original-se da reunião (b) ramus principalis dexter com o ramus principalis sinister. Comportamemto semellamte foi verificado, mais freqüentemente. tanto em cavalos S.R.D. (79.6\%). como em P.S.I. (36.7\%), respectivamente por FFERRIRA (1974) STOPIGI.IA el al." (1986). Resultados similares lambém já foram registrados no muar (80\%). por FERREIRA ol al. ${ }^{\text {.1.5. }}$ (1976): e em todas as peças estudadas. lante na zebra. por D'ERRICO et al.' ( 198.3 ). yuamto no jumento nordestino por PRADA et al. (1982). Nos equïideos, outras modalidadés de associação foram vistals, na composição doductus choledocus como a triconfluência, envolvendo o ramus principalis dexter, oramus principalis sinister coramus lobi quadrati. verificada em 30\% das glândulas nos P.S.I. (STOPICiLIA et al. $\left.{ }^{10}, 1986\right) ; 6,7 \%$ dos rigados nos cavalos S.R.D. (FERREIRA2. 1974) e 5\% das preparaçós nos muares (FERREIRA et al. "t.5. 1976).

O ramus principalis dexter é formado pelosramus ventralis lobi dextri. ramus medius lobi sinister, ramus dorsalis lobi 
MIGLINO. M.A.: SOULA. W.M.; DIDIO. I..J.A. Vius biliferas no tapir ou anta (Tapirus americanus). Braz. J. vet. Res. anim. Sci., São Paulo, v. 31 . n.2. p. $95-9.1994$.

dextri a ramus processi caudati, os quais conlluem de maneira distinta para comporem a aludida via: em um dos tígados. o segundo deles converge como último, compondo tronco comum. ao qual se associam. pela ordem. o ramus ventralis lobi dexter e o ranus distalis lobi dextri: no outro fígado, estes ductos associam-se dois a dois, ou seja, o primeiro ao segundo e o terceiro ao último, constituindo dois troncos que convergem para formar o ramus principalis sinister. Nos eqüinos S.R.D., FERREIRA² (1974) encontrou () ramus medius lobi dextri associando-se aoramus processi caudati em $70 \%$ das glândulas, resultado que foi observado em um dos tapires. No muar, esta modalidade de afluência dos ductos surge em .35\% dos lígados (FERREIRA et al. ${ }^{3,4,5}$, 1976) e nos animais P.S.I. em 26.7\% das dissecações (STOPIGI,IA et al."', 1986).

De outra parte, verificamos. na anta, que o ramus medius lobi sinister lateralis, oramus dorsalis lobi sinistri lateralis e o ramus lobi sinistri medialis tomam parte na formaçāo do ramus principalis sinister. Estes componentes já foram assinalados nos outros equídeos. em arranjos diversos. Particularmente no tapir. identificamos duas modalidades de associaçōes dos ductos: numa modalidade. o primciro ducto une-se ao segundo e o quarto conflui com o quinto lormando. assim, dois troncos que se associam compondo via comum à qual ven afluir o ramus lobo quadrati. Esta confluência foi patenteada, nos cavalos S.R.D. em apenas 3,3\% dos fígados (FERREIRA ${ }^{2}$, 1974) e em 5\% de muares (FERREIRA ct al. 5 , 1976), nāo revista nos animais P.S.J. (MARÇAL?, 1987). No outro fígado da anta. o ramus medius lobi sinistri lateralis reune-se com o ramus ventralis lobi sinistri lateralis. formando tronco, ao qual vêm ter. pela ordem, o ramus dorsalis lobi sinistri lateralis, o ramus lobi sinistri medialis c o ramus lobi quadrati. Arranjo idêntico a este foi visılo nos equiinos S.R.D. en 13.3\% dos casos (FERREIRA2. 1974), nos muares em 5\% (FERREIRA et al. $\left.{ }^{5} .1976\right)$ e nos equiinos P.S.I. em 6.7\% dos fígados (MARÇAL ${ }^{7}$ 1987).

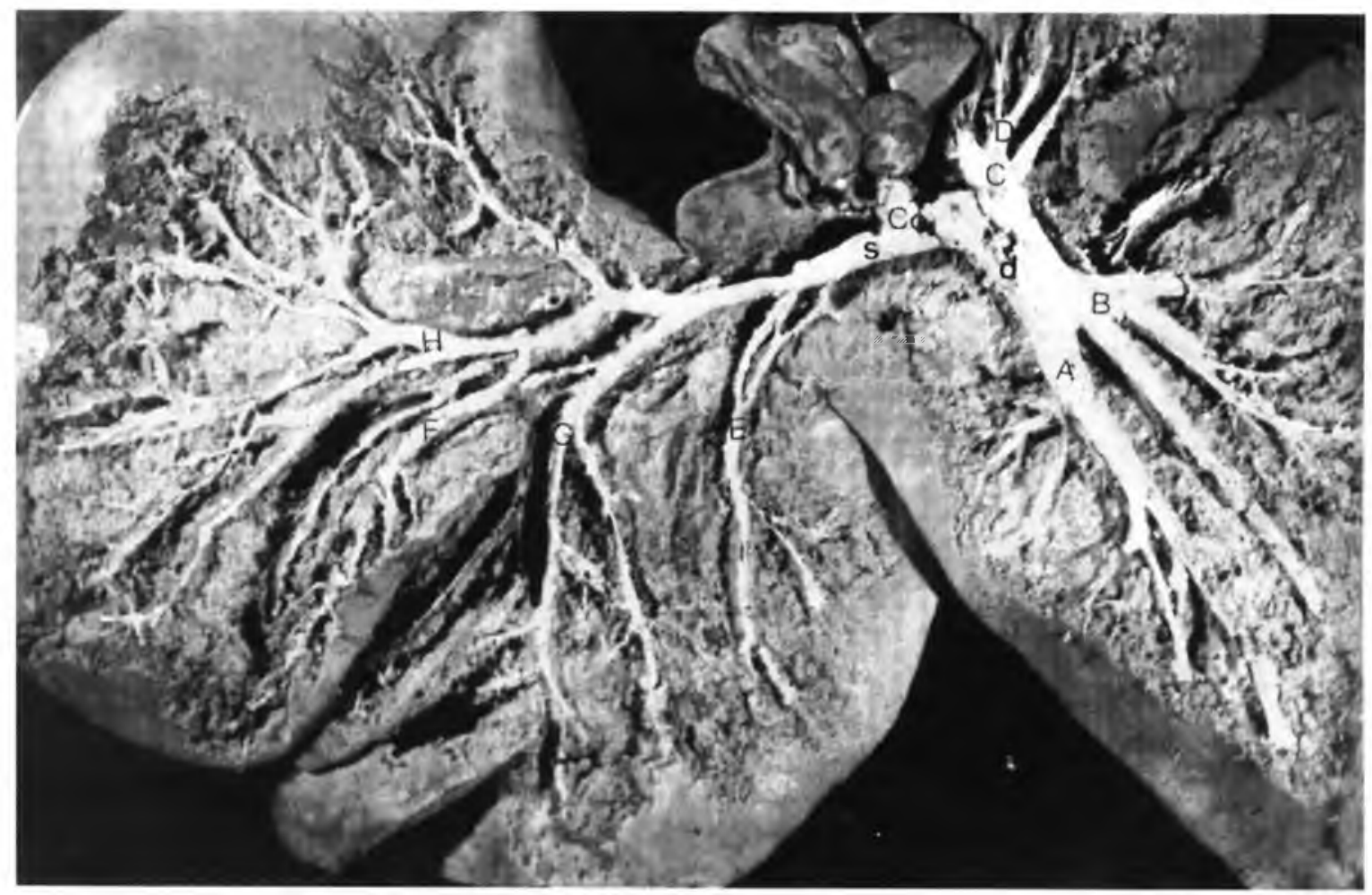

FIGURA I

Fotografia da face visceral do fígado de tapir (Tapirus americanus), macho, adulto, após dissecação, para mostrar a distribuição das vias bilíferas (injetadas com Neoprene de cor verde). Notam-se os 3 lobos do fígado: esquerdo. intermédio e direito, incompletamente separados por fissuras.

\section{I.EGENDA}

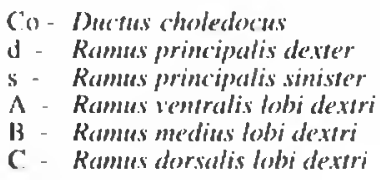

D) - Rarmus processi caudati

E. - Ramus hobi quadrati

F: - Ramus ventralis hobi sinistri lateralis

G - Ramus fobi simistrimedialis

H - Ramus medius tobi sinistri lateralis

1 - Ramus dorsatis tobi sinisuri lateralis 
MIGLINO, M.A.; SOUZA, W.M.; DIDIO, L.J.A. Vias bilíferas no tapir ou anta (Tapirus americanus). Braz. J. vet. Res. anim. Sci., São Paulo, v. 31 , n.2. p. $95-9,1994$

Estes ductos nominados únicos, tributários tanto do ramus principalis dexter como do ramus principalis sinister, já foram identificados em outros mamíferos deste grupo de animais, tais como: zebra (D'ERRICO et al.' , 1983) e jumento nordestino (PRADA et al. ${ }^{8}, 1983$ ). No tapir, o ramus principalis dexter, após constituído, năo possui tributários nos 2 fígados por nós estudados, mas FERREIRA² (1974) encontrou tributários na maioria das vezes $(63,3 \%)$ no cavalo S.R.D. e resultado idêntico foi também visto no P.S.I. (STOPIGLIA et al. ${ }^{10}, 1986$ ). O ramus principalis sinister na anta recebe afluentes inominados oriundos, simultaneamente, do lobus caudatus (pars supraportalis) e do lobus quadratus, sendo dois da primeira origem e um da segunda ( 1 vez) e um apenas do lobus quadratus ( 1 vez). Tanto FERREIRA2 (1974), nos cavalos S.R.D., quanto MARÇAL ${ }^{7}$ (1987), nos cavalos P.S.I., assinalam, neste particular, resultados semelhantes entre si, isto é, $50 \%$ e $46,6 \%$ respectivamente.

\section{CONCLUSÕES}

1. No Tapirus americanus, o ductus choledocus originase a partir da confluência do ramus principalis dexter e do ramus principalis sinister.
2. O ramus principalis dexter apresenta conno (onsulumtes constantes, o ramus ventralis lobi dextri, o ramus medius lobi dextri, o ramus dorsalis lobi dextri e o ramus processi caudati, os quais convergem, segundo duas modalidades, uma em cada fígado.

3. O ramus principalis sinister mostra, como componentes, o ramus medius lobi sinistri lateralis, o ramus dorsalis lobi sinistri lateralis, o ramus quadrati, o ramus ventralis lobi sinistri lateralis e o ramus lobi sinistri medialis; que se agrupam com dois diferentes arranjos, um em cada fígado.

4. O ramus principalis dexter não possui afluentes. O ramus principalis sinister possui afluentes provenientes do lobus caudatus (pars supraportalis) e do lobus quadratus.

\section{SUMMARY}

The biliary tree of two adult, male, tapirs (Tapirus americanus) was injected with latex. After the fixation of the liver with $10 \%$ formaldehyde solution the specimens were dissected and photographed. The ductus cloledocus is formed by the confluence of the ramus principalis dexter and sinister, since this species does not possess a vesica fellea. The ramus principalis dexter is made up of the ramus ventralis lobi dextri, ramus medius lobi dextri, ramus dorsalis lobi dextri and ramus processi caudati, which join each other under several artangements. The same occur with the ramus principalis sinister, formed by the ramus medius lobi sinistri lateralis, ramus dorsalis lobi sinistri lateralis, ramus lobi quadrati, ramus ventralis lobi sinistri lateralis and ramus lobi sinistri medialis.

UNITERMS: Liver; Tapirus; Bile ducts

\section{REFERÊNCIAS BIBLIOGRÁFICAS}

01-D`ERRICO, A.A.; PRADA, I.L.S.; FERNANDES FILHO, A. Contribuição ao estudo das vias bilíferas na zebra (Equus zebra L. 1758). In: SEMANA DE MEDICINA VETERINÁRIA, 2., São Paulo, 1983. Anais. p.7.

02-FERREIRA, N. Contribuição ao estudo das vias bilíferas intra e extra-hepáticas no Equus caballus. Sistematização doramus principalis dexter; ramus principalis sinister e do ductus choledocus. Rev. Fac. Med. Vet. Zootec. Univ. S. Paulo, v.11, p.201-16, 1974.

03-FERREIRA, N.; D'ERRICO, A.A.; FERNANDES FILHO, A.; BORELLI, V. Contribuição ao estudo das vias bilíferas extra-hepáticas no muar (Equus caballus $\mathrm{X}$ Equus asinus). In: CONGRESSO BRASILEIRO DE ANATOMIA, 9.; CONGRESSO LUSO BRASILEIRO DE ANATOMIA, 2., Niterói. 1976. Anais. p.48.

04-FERREIRA, N.; D'ERRICO, A.A.; FERNANDES FILHO, A.; BORELLI, V. Contribuição ao estudo das vias bilíferas intra-hepáticas no muar (Equus caballus XEquus asinus). Sistematização doramus principalis dexter. In: CONGRESSO BRASILEIRO DE ANATOMIA, 9.; CONGRESSO LUSO BRASILEIRO DE ANATOMIA, 2., Niterói, 1976. Anais. p.77. 
-FERREIRA, N.; D'ERRICO, A.A.; FFRNANDES FILHO. A.: BORELLI. V. Contribuição ao estudo das vias bilíferas intra-hepáticas no muar (Equus caballus $X$ Equus asinus). Sistematização doramus principalis sinister. In: CONGRESSO BRASILEIRO DE ANATOMIA. 9.: CONGRESSO LUSO BRASILEIRO DE ANATOMIA, 2., Niterói, 1976. Anais. p.48.

-JABLA.V-PANTIC, O. Characteristics and comparative ratios of intrahepatic bilc duct in domestic animais. Acta Vet., Bcograd, v.13, p.3-4, 1963.

-MARÇAL. A.V.Contribuição ao estudo das vias bilíferas em equinos da raça Puro Sangue Inglês. Sistematização do ramus principalis sinister. São Paulo, 1987. Dissertação (Mestrado) - Faculdade de Medicina Veterinária e Zootecia, Universidade de São Paulo.

.PRADA, I.L.S.; FEITOSA FILHO, E.; BORELLI, V.; FERNANDES FILHO. A. Contribuição ao estudo das vias bilíferas intra e extra-hepáticas no jumento nor- destino. In: SEMANA DE MEDICINA VETERINÁRIA, 1., São Paulo, 1983. Anais. p.77.

09-SCHUMMER, A.; NICKEL, R. The viscera of domestic mammals. In: NICKEL, R.; SCHUMMER, A.; SEIFERLE, E.; SACK. W.O. Textbook of the anatomy of the domestic animals. Berlim, Paul Parcy, 1979.

10-STOPIGLIA, A.J.; FERNANDES FILHO, A.; FERREIRA, N. Contribuição ao estudo das vias bilíferas intra e extra-hepáticas em cavalos Puro Sangue Inglês. Sistematização do ductus choledocus e ramus principalis dexter. Rev. Fac. Med. Vet. Zootec. Univ. S. Paulo. v.23, n.2, p.103-13, 1986.

Recebido para publicação em $05 / 04 / 93$ Aprovado para publicação en 19/10/93 